\title{
Efeito da adição de pozolana na resistividade elétrica superficial do concreto
}

\author{
Effect of pozzolan addition on surface electrical resistivity of concrete
}

\section{Ronaldo Alves Medeiros-Junior 1 (D) orcid.org/0000-0003-4879-4869 \\ Patrícia Schnepper Gans ${ }^{1}$ (D)orcid.org/0000-0002-8560-1328}

\author{
1 Programa de Pós-Graduação em Engenharia de Construção Civil, Departamento de Construção Civil, Universidade Federal do Paraná, \\ Curtiba, Brasil. \\ E-mail do autor principal: Ronaldo Alves de Medeiros-Junior ronaldodemedeirosjr@yahoo.com.br
}

Resumo

A resistividade elétrica superficial é um parâmetro interessante para controlar a vida útil de estruturas de concreto, principalmente devido à praticidade de execução do ensaio. O objetivo deste artigo é comparar a resistividade elétrica superficial (RES) de concretos com dois diferentes tipos de cimento: um com adição de pozolana (CP IV) e outro sem adição mineral (CP V). A RES foi medida pelo método de Wenner. Foi utilizado o mesmo traço 1:1,4:2,1 (cimento: areia: brita) e a mesma relação água/cimento de 0,50 para os dois tipos de concreto investigados. Os resultados foram analisados nas idades de 28, 60, 90, 120, 150 e 180 dias. Para todas as amostras foi identificado um aumento da RES com o tempo de ensaio. Porém, no cimento com adição de pozolana, o fator idade do concreto elevou-se $93 \%$ em relação ao fator idade das amostras de CP V. A RES foi muito superior para o concreto de cimento com adição pozolânica em todas as idades, atingindo valor 4,9 vezes maior que amostra de concreto com cimento CP V aos 180 dias.

Palavras-Chave: Concreto; Resistividade elétrica superficial; Pozolana.

\begin{abstract}
The surface electrical resistivity is an interesting parameter to control the useful life of concrete structures, mainly due to the practicality of the test. The aim of this study was to evaluate the change in surface electrical resistivity (SER) of concretes produced with two different types of cement: a cement with pozzolan (CP IV) and a cement without addition (CP V). It was used the same proportion between materials and the same water/cement ratio for the samples. The SER was measured using the Wenner Method for 28, 60, 90, 120, 150 and 180 days. The results indicated that the SER increased with time for both types of cement, although the age factor of the concrete with CP IV was 93\% higher. This concrete had better results in every age and it was almost five times greater for 180 days when comparing with samples produced with CP V.
\end{abstract}

Key-words: Concrete; Surface electrical resistivity; Pozzolan. 


\section{Introdução}

As normas em vigor de dimensionamento de estruturas de concreto têm apresentado dificuldade em garantir durabilidade suficiente em ambientes agressivos. Fato confirmado pelo número de manifestações patológicas observadas nas estruturas, resultado do envelhecimento precoce das construções. Ao integrar o conceito de durabilidade ao projetar estruturas de concreto, é necessário desenvolver métodos de predição e monitoramento de vida útil [1].

Relatórios técnicos de países ibero-americanos classificam a corrosão das armaduras como um dos processos de degradação que mais comprometem a vida útil das estruturas de concreto, juntamente com a fissuração e a deformação por carga [2]. A corrosão das armaduras é um processo eletroquímico já muito discutido da literatura. A deterioração causada pela interação fisico-química entre a armadura e o meio em que está inserida acarreta alterações prejudiciais indesejadas, como redução da área de aço, redução da tensão de escoamento do aço, fissuração do concreto de cobrimento decorrente dos produtos expansivos originados das reações químicas e perda da aderência entre concreto e armadura, o que torna a estrutura inadequada para o uso [3][4]. A resistividade elétrica é uma propriedade dos materiais e caracteriza a dificuldade dos íons se movimentarem no concreto. Essa propriedade é o inverso da condutibilidade elétrica, e controla o fluxo de íons difundidos no interior do concreto, atráves da solução aquosa presente em seus poros [5].

Os cloretos são os agentes agressivos responsáveis por iniciar a corrosão mais documentados na literatura [5][6]. A difusão desses íons no interior do concreto pode despassivar a armadura e desencadear o processo corrosivo, afetando, principalmente, estruturas em zona de agressividade marinha [7]. O processo eletroquímico que gera a corrosão pode ser controlado, principalmente, pela resistividade elétrica e pela disponibilidade de oxigênio às barras de armadura [8]. Portanto, a resistividade elétrica é um indicador de durabilidade. Quanto maior seu valor, ou seja, menor a mobilidade de íons no concreto, menor é a tendência a ocorrer o processo corrosivo.

A proporção de materiais na mistura, a relação água/cimento e a utilização de adições minerais e de aditivos têm capacidade de alterar as propriedades elétricas do concreto, pois modificam tanto a 95 microestrutura da matriz, quanto as características da solução presente nos poros [9].

As adições minerais são insumos da Construção Civil que possuem baixo custo e substituem o clínquer, um material nobre que necessita de muita energia para ser obtido. Portanto, a utilização das adições minerais no concreto decorre de vários fatores, desde econômicos até ecológicos. As adições podem ser caracterizadas como inertes (apenas efeito filler), cimentantes (escória de alto forno) e pozolânicas (cinza volante, sílica ativa, metacaulim, entre outras) [10].

Gans et al. [11] estudaram o efeito da escória de alto forno na resistividade elétrica superficial do concreto. Estes autores mostraram que o crescimento da resistividade elétrica no tempo é maior para o cimento com adição de escória em comparação com um cimento CP V. Além disso, a resisitivadade foi muito superior para o concreto com cimento de escória de alto forno, atingindo valor 9,7 vezes maior que amostra de concreto com cimento CP $\vee$ aos 180 dias.

A pozolana é um material silicoso ou sílicoaluminoso que, quando finamente moído e na presença de água, reage com o hidróxido de cálcio presente na matriz do concreto, em temperatura ambiente, formando gel C-S-H [12]. Portanto, ao misturar adições pozolânicas ao clínquer, ocorrem mudanças na matriz do concreto decorrentes da reação química entre os componentes do cimento e a água, e podem impactar nas propriedades elétricas do concreto.

Dessa forma, o objetivo deste trabalho é avaliar o efeito da adição da pozolana presente no cimento na resistividade elétrica do concreto. A escolha do estudo da pozolana está relacionada a grande utilização deste material no Brasil, principalmente na região Sul.

\section{Programa Experimental}

Para que fosse possível comparar o efeito da adição de pozolana na resistividade elétrica superficial do concreto, foram selecionados dois tipos de cimento para produção dos corpos de prova: cimento Portland pozolanico, classe 32 (CP IV 32), e cimento Portland de alta resistência inicial (CP V ARI). O CP IV 32 possui adição de até $50 \%$ de pozolana em sua composição, já o CP V é basicamente formado por clínquer e 
sulfatos de cálcio. A pozolana usada no cimento CP IV é a cinza volante. Destaca-se que esse trabalho foi desenvolvido na região Sudeste do Brasil.

As caraterísticas químicas dos dois cimentos, segundo os limites das normas NBR 5736 [12] e NBR 5733 [13], estão apresentadas na Tabela 1.

Tabela 1: Propriedades químicas: Limites de normas brasileiras.

\begin{tabular}{l|l|l}
\hline $\begin{array}{l}\text { Propriedades químicas } \\
\text { (unidade: \% em volume) }\end{array}$ & CP IV 32 & CP V \\
\hline NORMA ABNT & NBR 5736 & NBR 5733 \\
\hline Clínquer + sulfatos de cálcio & $85-45$ & $100-95$ \\
\hline Material pozolânico & $15-50$ & - \\
\hline Material carbonático & $0-5$ & $0-5$ \\
\hline Óxido de magnésio (MgO) & 6,5 & 6,5 \\
\hline Trióxido de enxofre (SO3) & 4,0 & - \\
\hline Perda ao fogo & 4,5 & 4,5 \\
\hline Resíduos Insolúveis & - & 1,0 \\
\hline Anidrido Carbônico (CO2) & 3,0 & 3,0 \\
\hline
\end{tabular}

Fonte: NBR 5736 [12] e NBR 5733 [13]

Foram utilizados agregados miúdo e graúdo disponíveis comercialmente na cidade de São José dos Campos. Como agregado miúdo, foi utilizada areia natural proveniente de rio, com dimensão máxima característica de 2,36mm e módulo de finura 2,6. 0 agregado graúdo utilizado foi brita comercial com dimensão máxima de 9,5 mm e módulo de finura 6,5.

Foi fixado o traço $1: 1,4: 2,1$ (cimento: areia: brita), em materiais secos, e a relação água/cimento de 0,50 para fabricação de todos os corpos de prova. Portanto, a relação entre cimento e agregados usada foi $1: 3,5$, com consumo de cimento de $474 \mathrm{~kg} / \mathrm{m}^{3}$.

Baseado na experiência de ensaios discutida por Lencioni [14], os corpos de prova tiveram formato cúbico de $250 \times 250 \times 250 \mathrm{~mm}$. Foram utilizadas formas de madeira, e o adensamento foi feito com vibrador de imersão em 3 camadas. Foram moldados 4 corpos de prova para cada tipo de concreto, somando ao todo 8 corpos de prova.

Os corpos de prova permaneceram com a superfície coberta e umedecida por 48 horas até o momento da desmoldagem, para evitar troca de água com a superfície. Após, ocorreu a cura saturada em câmara úmida (umidade relativa do ar igual ou maior a $95 \%$ ), durante um período de 28 dias. Então, foram 96 colocados em ambiente de laboratório até as idades de realização dos ensaios.

Foram realizadas medidas de resistividade elétrica superficial (RES) aos 28,60, 90, 120, 150 e 180 dias pelo Método de Wenner, ou método dos quatro pontos. Para tal, foi utilizado um equipamento que possui quatro eletrodos espaçados a igual distância ( $a$ $=0,05 \mathrm{~m}$ ). Ao ser posicionado sobre a superfície do concreto, uma corrente elétrica é aplicada entre os dois contatos externos e a diferença de potencial resultante é medida pelos dois contatos internos.

O valor da RES é determinado pela Equação (1) [15]. Um esquema desse procedimento é ilustrado na Figura 1.

$$
\rho=2 \pi a \frac{V}{I}
$$

Em que: $\rho=$ resistividade elétrica ( $\Omega . \mathrm{m}) ; \mathrm{a}=$ distância entre os eletrodos $(\mathrm{m}) ; \mathrm{V}=$ diferença de potencial (V) entre dois pontos; I = corrente resultante $(A)$ resultante.

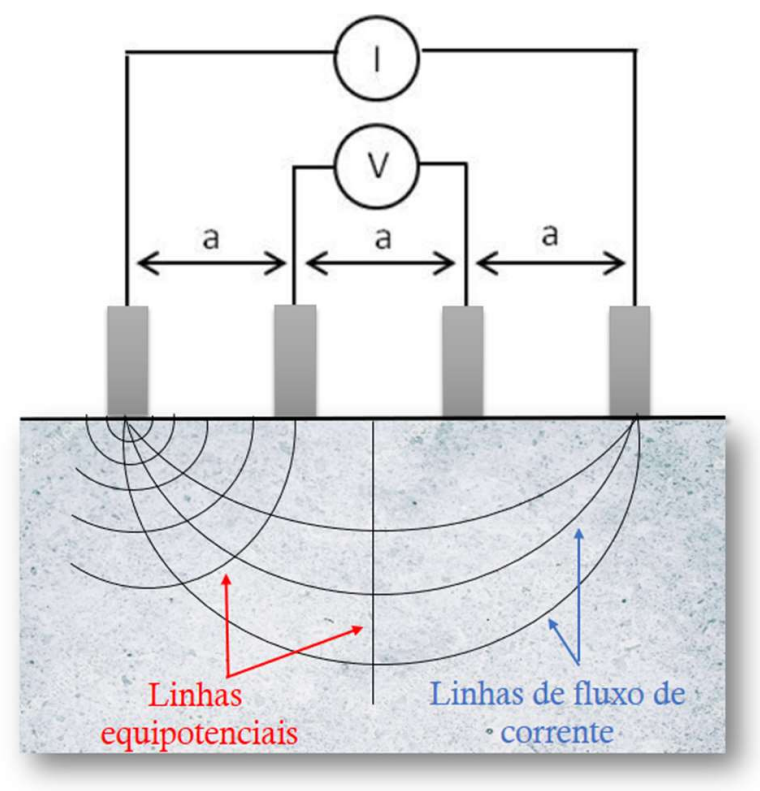

Figura 1: Esquema da Técnica de Wenner para medida da RES do concreto.

Fonte: Gowers e Millard (1999) traduzido por MedeirosJunior [7].

Com os dados de RES obtidos ao longo do tempo, foi possível calcular o fator idade $(q)$, através do

http: / / dx.doi.org/10.25286/repa.v2i3.692 
ajuste de potência da Equação (2). O fator idade mede a evolução da RES ao longo do tempo [1].

$$
\rho_{2}=\rho_{0} \cdot\left(\frac{t_{2}}{t_{o}}\right)^{q}
$$

Em que: $\rho_{2}=$ resistividade elétrica $(\Omega . \mathrm{cm})$ no tempo $\mathrm{t}_{2}$ (anos) considerado; $\rho_{0}=$ resistividade elétrica $(\Omega . \mathrm{cm})$ na idade (to, em anos) da primeira medição (usualmente igual a 28 dias de idade, após cura saturada do concreto); e q = fator idade.

\section{Resultados e Discussões}

Os resultados do ensaio de RES para as amostras contendo o cimento $\mathrm{CP} V$ e CP IV, respectivamente, para as idades de 28, 60, 90, 120, 150 e 180 dias estão apresentados nas Figuras 2 e 3 . Os resultados representam a média dos valores de RES de quatro corpos de prova para cada tipo de concreto investigado.

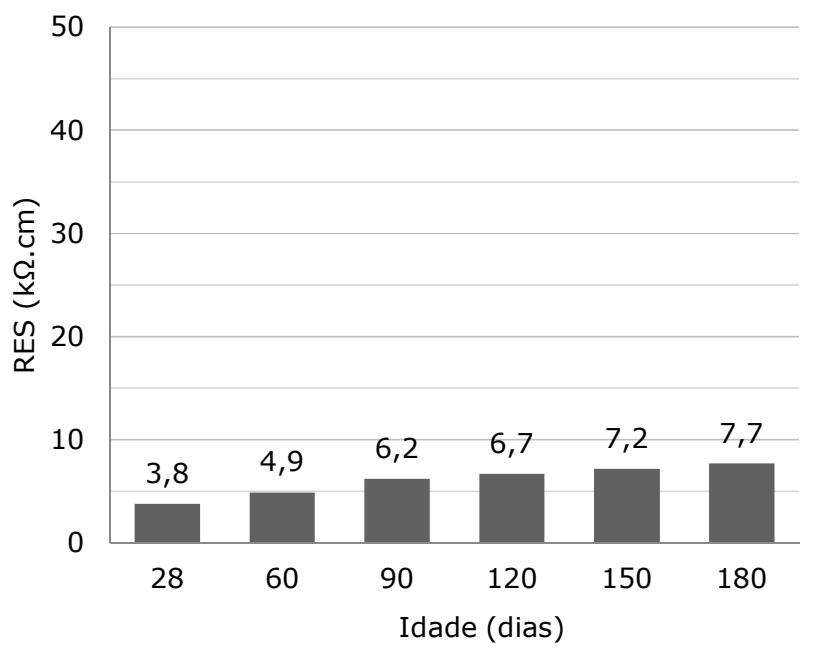

Figura 2: Resultados de resistividade elétrica superficial (RES) para as amostras de concretos com cimento CP V.

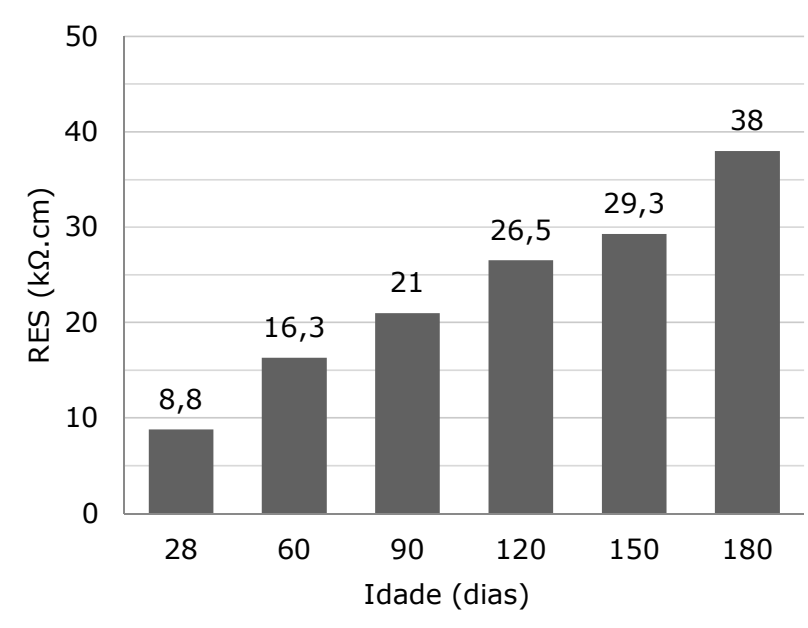

Figura 3: Resultados de resistividade elétrica superficial (RES) para as amostras de concretos com cimento CP IV.

Ao comparar os resultados das Figuras 2 e 3, é visto que os valores de RES das amostras de concreto com cimento CP IV são superiores aos valores obtidos para as amostras de concreto com cimento CP V, para todas as idades. Na primeira idade investigada, ou seja, aos 28 dias, a média da RES das amostras de CP IV foi 2,3 vezes maior que os resultados do cimento CP V. Já aos 180 dias, esse valor foi 4,9 vezes superior.

A finura dos cimentos também impacta nos resultados de resistividade elétrica. Para um cimento com uma mesma composição química, o cimento mais fino resulta em maior resisistividade elétrica superficial, visto que reage mais rápido. Contudo, ao comparar o cimento CP V e CP IV, com composição químicas distintas, o efeito da presença de pozolanas parece sobressair ao efeito da finura, que é maior para o cimento CP V [16][17]. Portanto, os resultados das Figuras 2 e 3 podem ser atribuídos à grande quantidade de adição de pozolana no cimento CP IV (cerca de 15 a 50\%, conforme Tabela 1).

A pozolana também promove um efeito filler, o qual aumenta a densidade do concreto, assim como a resistência da matriz e a sinuosidade capilar, além de reduzir a concentração iônica nos poros [18]. Tal fenômeno deve-se à mudança na cinética de hidratação do cimento quando são inseridas no sistema cimentício de partículas de dimensão similar ou inferior às partículas do clínquer, visto que essas geram pontos extras de nucleação, em que hidratos precipitam sobre a superfície desses grãos [19]. Portanto, ao refinar os poros da microestrutura do 
concreto, é diminuída a condutividade das amostras e, consequentemente, aumenta-se a RES.

A RES aumenta com o tempo devido à evolução das reações de hidratação e endurecimento do concreto, comportamento que também é observado nas Figuras 2 e 3 . O mesmo também foi identificado por Baweja et al. [20], CCAA [21], Andrade e D'Andréa [22], Presuel-Moreno et al. [23] e Medeiros-Junior e Lima [24], e pode ser explicado devido ao concreto apresentar maior condutibilidade elétrica nas primeiras horas. Ao misturar os componentes do concreto, forma-se uma pasta de cimento e água, e a água atua como condutor de íons dissolvidos no interior da massa do concreto.

Na Figura 4 está representado o ajuste de potência que foi feito para as amostras de cada cimento, a fim de encontrar o fator idade $q$. Esse ajuste representa a variação da RES com o tempo, em que to é a idade da primeira medida de ensaio, ou seja, 28 dias.

Ao comparar os valores de RES aos 28 e 180 dias, para as amostras de cimento $C P \quad V$ houve um crescimento de $102 \%$. Já para as amostras de cimento CP IV, o crescimento foi de 332\%. Logo, o cimento com adição de pozolana, além de apresentar maiores valores de RES para todas as idades, também apresentou maior evolução com o tempo. Cimentos com adições têm maior evolução da resistividade ao longo do tempo, porque as reações das adições são mais lentas, contudo mais graduais, em comparação com o cimento com a maior quantidade de clínquer [24].

Esse fenômeno também é notado pelos valores dos fatores idade. De acordo com o ajuste feito na Figura 4, foi encontrado um fator idade igual a 0,752 para o concreto com cimento CP IV, valor $93 \%$ maior ao fator idade do concreto com cimento CP V, igual a 0,388.

Resultados em concordância com os apresentados já foram obtidos na bibliografia. Em sua pesquisa, Ferreira e Jalali [25] obtiveram valores iniciais de resistividade elétrica maiores e maior taxa de crescimento para o concreto com cimento CEM IV, com adição de pozolana, em comparação ao CEM I, sem adições.

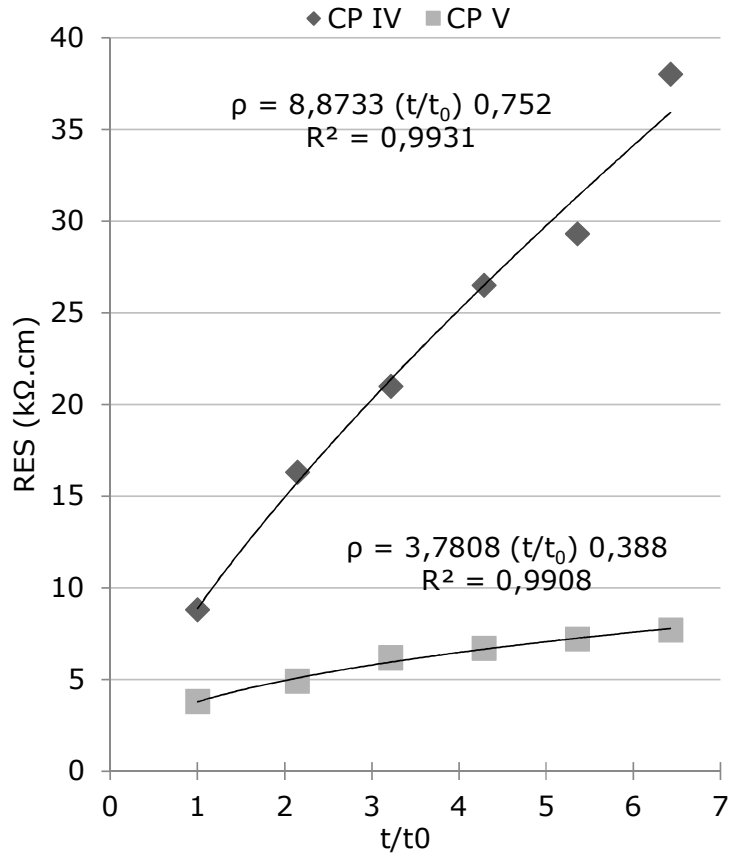

Figura 4: Evolução da RES no tempo para ambos os tipos de cimento.

Medeiros-Junior et al. [26] e Medeiros-Junior e Lima [27] também mediram a resistividade elétrica superficial em concretos de diferentes tipos de cimento e obtiveram maiores valores em concretos de cimento com adições, incluindo o cimento CP IV, quando comparados a concretos com CP V e CPII-F. Os autores afirmam que o efeito de refinamento dos poros e redução da porosidade causados pelas escórias e pozolanas ocasionou a redução da condutividade do concreto. Portanto, os cimentos com adições reduzem a mobilidade dos íons dentro da microestrutura do concreto.

Medeiros-Junior e Lima [24] demonstraram que as diferenças entre os valores de resistividade para diferentes tipos de cimento e afirmam que as adições minerais pozolânicas em cimentos tendem a consumir hidróxido de cálcio ao longo do tempo, produzindo mais gel C-S-H no concreto devido a reações pozolânicas. Tais reações diminuem a quantidade do íon $\mathrm{OH}^{-}$na solução dos poros, reduzindo a condutividade do concreto, e, consequentemente, contribuindo para aumentar a resistividade elétrica.

Ao observar o ajuste dos resultados da série contendo cimento CP V na Figura 4, também nota-se http: / / dx.doi.org/10.25286/repa.v2i3.692 
que há uma têndencia de estabilização da RES, em função do menor acréscimo no tempo. Já para a série contendo CP IV, não há sinal de estabilização no período de medição estabelecido.

\section{Conclusão}

As amostras de concreto com cimento CP IV, o qual contém adição pozolânica, apresentam maiores valores de RES para todas as idades investigadas. $\mathrm{Na}$ data mais avançada investigada nesse estudo, ou seja, 180 dias, a RES dessas amostras é 4,9 vezes maior que o valor das amostras de concreto com cimento CP V.

A RES também aumentou com o tempo de ensaio, resultado da hidratação e endurecimento do concreto. Para as amostras de concreto com cimento CP IV, a evolução com o tempo é maior, quando comparado às amostras de concreto com cimento CP V. O fator idade do concreto com adição mineral é $93 \%$ superior ao sem essa adição.

Conclui-se que a adição de pozolana na composição do cimento contribui significativamente para o aumento da resistividade elétrica superficial do concreto.

Para trabalhos futuros, sugere-se a investigação da estabilização da RES para cimentos com adição, ao monitorar essa propriedade para idades mais avançadas. Também pode-se recomendar a comparação da RES entre cimentos com adição pozolânica e cimentos com escória de alto forno.

\section{Agradecimentos}

À Universidade Federal do Paraná (UFPR), em especial ao Programa de Pós-Graduação em Engenharia de Construção Civil (PPGECC).

\section{Referências}

[1] ANDRADE, C.; D'ANDREA, R. Electrical resistivity as microstructural parameter for the modelling of service life of reinforced concrete structures. In: 2ND INTERNATIONAL SYMPOSIUM ON SERVICE LIFE DESIGN FOR INFRASTRUCTURE. Anais... p. 379-388. Delft, Holanda, 2010.

[2] RINCÓN, O. T. Durability of concrete structures: DURACON, an Iberoamerican project.
Preliminary results Building and Environment. v. 41, p. 952-962, 2006.

[3] GENTIL, V. Corrosão, 3. ed. Editora LTC. Rio de Janeiro, 2006.

[4] PELLIZZER, G. P. Análise mecânica e probabilística da corrosão de armaduras de estruturas de concreto armado submetidas à penetração de cloretos. 2015. Dissertação (Mestrado) - Escola de Engenharia de São Carlos. São Carlos.

[5] HELENE, P. Contribuição ao Estudo da Corrosão em Armaduras de Concreto Armado. 1993. Tese (Livre Docência) - Escola Politécnica da Universidade de São Paulo. São Paulo.

[6] CASCUDO, O. O controle da corrosão de armaduras em concreto: inspeção técnicas eletroquímicas. Editora da UFG Goiânia. Goiás, 1997.

[7] MEDEIROS-JUNIOR, R. A. Estudo da resistividade do concreto para proposta de modelagem de vida útil - Corrosão das armaduras devido à penetração de cloretos. 2014. Tese (Doutorado) - Instituto Tecnológico de Aeronáutica. São José dos Campos, São Paulo.

[8] LENCIONI, J. W; LIMA, M. G. Principais fatores intervenientes nas medidas de resistividade elétrica superficial do concreto-Estado-da-Arte. In: VI CONGRESSO INTERNACIONAL SOBRE PATOLOGIA E REABILITAÇÃO DE ESTRUTURAS. Anais... Córdoba, Argentina, 2010.

[9] POLDER, R. B. Test methods for on site measurement of resistivity of concrete-a RILEM TC-154 technical recommendation. Construction and building materials, v. 15, n. 2, p. 125-131, 2001.

[10] MEHTA P. K., MONTEIRO P. J. M. Concreto: estrutura, propriedades e materiais, São Paulo: Editora Pini, p.674, 2006.

[11] GANS, P. S.; MEDEIROS-JUNIOR, R. A.; LIMA, M. G. Efeito da escória de alto forno na resistividade elétrica superficial do concreto. In: $2^{\circ}$ SIMPÓSIO PARANAENSE DE PATOLOGIA DAS CONSTRUÇÕES. Anais... Curitiba, Brasil, $2017 .$.

[12] ASSOCIAÇÃO BRASILEIRA DE NORMAS TÉCNICAS (ABNT). NBR-5736: Cimento Portland Pozolânico. Rio de Janeiro, 1999. 
[13] ASSOCIAÇÃO BRASILEIRA DE NORMAS TÉCNICAS (ABNT). NBR-5733: Cimento Portland de alta resistência inicial. Rio de Janeiro, 1991.

[14] LENCIONI, J. W. Estudos sobre resistividade elétrica superficial em concreto: análise e quantificação de parâmetros intervenientes nos ensaios. 2011. Tese (doutorado) - Programa de Pós-Graduação em Engenharia de Infraestrutura Aeronáutica. Instituto Tecnológico de Aeronáutica - ITA. São José dos Campos, São Paulo.

[15] HALLIDAY, D.; RESNICK, R. Fundamentos da física. Eletromagnetismo. $8^{a}$ ed. Rio de janeiro: Editora LCT, v. 3, 2009.

[16] VAN NOORT, R.; HUNGER, M.; SPIESZ, P. Long-term chloride migration coefficient in slag cement-based concrete and resistivity as an alternative test method. Construction and Building Materials, v. 115, p. 746-759, 2016.

[17] GIRARDI, R. Estudo da variabilidade do cimento Portland que abasteceu o mercado do Rio Grande do Sul no período de 1992 a 2012. 2014. Dissertação (Mestrado) - Universidade Federal do Rio Grande do Sul, Porto Alegre.

[18] BIJEN, ]. Benefits of slag and fly ash. Construction and building materials, v. 10, n. 5, p. 309-314, 1996.

[19] LAWRENCE, P.; CYR, M.; RINGOT, E. Mineral admixtures in mortars: effect of inert materials on short-term hydration. Cement and concrete research, v. 33, n. 12, p. 1939-1947, 2003.

[20] BAWEJA, D.; ROPER, H.; SIRIVIVATNANON, V. Quantitative descriptions of steel corrosion in concrete using resistivity and anodic polarisation data. In.: 4TH CANMET/ACI INTERNATIONAL CONFERENCE ON DURABILITY OF CONCRETE.

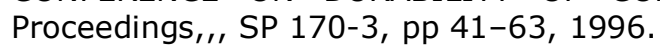

[21] CEMENT CONCRETE \& AGGREGATES AUSTRALIA (CCAA). Chloride Resistance of Concrete. Report, 2009.

[22] ANDRADE, C.; D'ANDRÉA, R. La resistividad eléctrica como parámetro de control del hormigón y de su durabilidad. Revista ALCONPAT, v. 1, n. 2, p. 93-101, 2011.

[23] PRESUEL-MORENO, F.; WU, Y.-Y.; LIU, Y. Effect of curing regime on concrete resistivity and aging factor over time. Construction and Building Materials, v. 48, p. 874-882, 2013.
[24] MEDEIROS-JUNIOR, R. A.; LIMA, M. G. Electrical resistivity of unsaturated concrete using different types of cement. Construction and Building Materials, v. 107, p. 11-16, 2016.

[25] FERREIRA, R. M.; JALALI, S. NDT measurements for the prediction of 28-day compressive strength. NDT \& E International, $v$. 43, n. 2, p. 55-61, 2010.

[26] MEDEIROS-JUNIOR, R. A.; LIMA, M. G.; MEDEIROS, M. H. F.; REAL, L. V. Investigação da resistência à compressão e da resistividade elétrica de concretos com diferentes tipos de cimento. Revista ALCONPAT, v. 4, n. 2, p. 116-132, 2014.

[27] MEDEIROS-JUNIOR, R. A.; LIMA, M. G. Variação da resistividade elétrica do concreto com o formato de corpos de prova para diferentes tipos de cimento. Revista de Engenharia e Tecnologia, v. 7, p. 1-8, 2015. 